T. Ishikawa

Nagoya Math. J.

Vol. 118 (1990), 195-202

\title{
SUPER CONGRUENCE FOR THE APÉRY NUMBERS
}

\author{
TSUNEO ISHIKAWA
}

\section{§. Introduction}

Let, for any $n \geq 0$,

$$
a(n)=\sum_{k=0}^{n}\left(\begin{array}{l}
n \\
k
\end{array}\right)^{2}\left(\begin{array}{c}
n+k \\
k
\end{array}\right), \quad u(n)=\sum_{k=0}^{n}\left(\begin{array}{l}
n \\
k
\end{array}\right)^{2}\left(\begin{array}{c}
n+k \\
k
\end{array}\right)^{2} .
$$

R. Apéry's proof of the irrationality of $\zeta(2)$ and $\zeta(3)$ made use of these numbers (see [10]). As a result, many properties of the Apéry numbers were found (see [1]-[9]). In particular, Beukers and Stienstra showed the interesting congruence (see [11, Theorem 13.1]).

Theorem 1 (Beukers and Stienstra). Let $p \geq 3$ be a prime, and write

$$
\sum_{n=1}^{\infty} \lambda_{n} q^{n}=q \prod_{n=0}^{\infty}\left(1-q^{4 n}\right)^{6}
$$

Let $m, r \in N, m$ odd, then we have

$$
\begin{aligned}
& a\left(\frac{m p^{r}-1}{2}\right)-\lambda_{p} a\left(\frac{m p^{r-1}-1}{2}\right)+(-1)^{(p-1) / 2} p^{2} a\left(\frac{m p^{r-2}-1}{2}\right) \\
& \quad \equiv 0 \bmod p^{r} .
\end{aligned}
$$

Moreover they conjectured that congruence (2) holds $\bmod p^{2 r}$ if $p \geq 5$, and they called these congruences super congruences in [4] and [11].

In this paper we shall prove the conjecture for $r=1$.

TheOREM 2. Let $p \geq 5$ be a prime and $m \in N, m$ odd, then we have

$$
a\left(\frac{m p-1}{2}\right)-\lambda_{p} a\left(\frac{m-1}{2}\right) \equiv 0 \bmod p^{2} .
$$

F. Beukers informed me that L. Van Hamme proved the case of $p \equiv$ 1 mod 4 using properties of the $p$-adic gamma function (see [7]). We prove the general case involving $p \equiv 3 \bmod 4$ by entirely different method. Our

Received April 15, 1989. 
method is applicable to super congruences of other numbers such as $u(n)$ (see [8]).

\section{$\S 1$. Congruence of $a(n)$}

The numbers $a(n)$ satisfy the recurrence

$$
(n+1)^{2} a(n+1)=\left(11 n^{2}+11 n+3\right) a(n)+n^{2} a(n-1) \quad n \geq 1 .
$$

We know the following result. Let $p$ be an odd prime, and $m \geq 0$, then

$$
\begin{array}{ll}
a(m p) \equiv a(m) & \bmod p^{2}, \\
a(p-1) \equiv 1 & \bmod p^{2} .
\end{array}
$$

By (3), (4) and (5), we have $a(p-2) \equiv-3+5 p \bmod p^{2}, a(p+1) \equiv 9+$ $15 p \bmod p^{2}$.

Proposition 1. Let $m \geq 0, n \geq 0$ and $m+n=p-1$. Then

$$
a(m) \equiv(-1)^{m} a(n) \quad \bmod p .
$$

Proof. We proceed by induction on $m$ to show that $a(m) \equiv$ $(-1)^{m} a(p-m-1) \bmod p$. From the above result, $a(0) \equiv a(p-1) \equiv 1 \bmod p$ and $a(1) \equiv-a(p-2) \equiv 3 \bmod p$. Let $0<m<p-1$. From the recurrence (3),

$$
\begin{aligned}
& (m+1)^{2} a(m+1) \\
& \quad=\left(11 m^{2}+11 m+3\right) a(m)+m^{2} a(m-1) \\
& \quad \equiv\left\{11(p-m)^{2}-11(p-m)+3\right\} a(m)+(p-m)^{2} a(m-1) \\
& \quad \equiv\left\{\begin{array}{rr}
-\left\{11(p-m)^{2}-11(p-m)+3\right\} a(p-m-1)+(p-m)^{2} a(p-m) \\
\text { if } m: \text { odd } \\
\left\{11(p-m)^{2}-11(p-m)+3\right\} a(p-m-1)-(p-m)^{2} a(p-m)
\end{array}\right. \\
& \quad \equiv\left\{\begin{array}{rr}
(m+1)^{2} a(p-m-2) & \text { if } m: \text { odd } \quad \bmod p . \\
-(m+1)^{2} a(p-m-2) & \text { if } m: \text { even }
\end{array}\right.
\end{aligned}
$$

Proposition 2. For all primes $p, n \geq 0$ and $0 \leq m \leq p-1$, we have

$$
a(n p+m) \equiv a(m) a(n) \quad \bmod p .
$$

Proof. This congruence follows from the similar method of the proof of [6, Theorem 1].

Q.E.D. 


\section{§2. Congruence of $\boldsymbol{b}(\boldsymbol{n})$}

Let $b(n)=0$ and, for any $n \geq 1$,

$b(n)=\sum_{k=1}^{n}\left(\begin{array}{c}n \\ k\end{array}\right)^{2}\left(\begin{array}{c}n+k \\ k\end{array}\right)\left[\frac{2}{n-k+1}+\cdots+\frac{2}{n}+\frac{1}{n+1}+\cdots+\frac{1}{n+k}\right]$.

These numbers are (differential) of $a(n)$ and they take important parts in the congruence of $\bmod p^{2}$ as shown in [6, Theorem 4].

Proposition 3. The numbers $b(n)$ satisfy the recurrence

$$
\begin{aligned}
(n+1)^{2} b(n+1)= & \left(11 n^{2}+11 n+3\right) b(n)+n^{2} b(n-1) \\
& -2(n+1) a(n+1)+11(2 n+1) a(n)+2 n a(n-1),
\end{aligned}
$$

and for all primes $p \geq 3, n \geq 0$ and $0 \leq m \leq p-1$, we have

$$
a(n p+m) \equiv\{a(m)+p n b(m)\} a(n) \quad \bmod p^{2} .
$$

Proof. Let

$$
\begin{aligned}
B_{n, k}= & \left(k^{2}+3(2 n+1) k-11 n^{2}-9 n-2\right)\left(\begin{array}{c}
n \\
k
\end{array}\right)^{2}\left(\begin{array}{c}
n+k \\
k
\end{array}\right) H_{n, k} \\
& +(6 k-22 n-9)\left(\begin{array}{c}
n \\
k
\end{array}\right)^{2}\left(\begin{array}{c}
n+k \\
k
\end{array}\right),
\end{aligned}
$$

and

$$
H_{n, k}=\frac{2}{n-k+1}+\cdots+\frac{2}{n}+\frac{1}{n+1}+\cdots+\frac{1}{n+k},
$$

then we have

$$
\begin{aligned}
B_{n, k}-B_{n, k-1}= & (n+1)^{2}\left(\begin{array}{c}
n+1 \\
k
\end{array}\right)^{2}\left(\begin{array}{c}
n+1+k \\
k
\end{array}\right) H_{n+1, k} \\
& -\left(11 n^{2}+11 n+3\right)\left(\begin{array}{c}
n \\
k
\end{array}\right)^{2}\left(\begin{array}{c}
n+k \\
k
\end{array}\right) H_{n, k} \\
& -n^{2}\left(\begin{array}{c}
n-1 \\
k
\end{array}\right)^{2}\left(\begin{array}{c}
n-1+k \\
k
\end{array}\right) H_{n-1, k} \\
& +2(n+1)\left(\begin{array}{c}
n+1 \\
k
\end{array}\right)^{2}\left(\begin{array}{c}
n+1+k \\
k
\end{array}\right) \\
& -11(2 n+1)\left(\begin{array}{c}
n \\
k
\end{array}\right)^{2}\left(\begin{array}{c}
n+k \\
k
\end{array}\right)-2 n\left(\begin{array}{c}
n-1 \\
k
\end{array}\right)^{2}\left(\begin{array}{c}
n-1+k \\
k
\end{array}\right) .
\end{aligned}
$$

Taking summation from 1 to $n+1$ on $k$, recurrence (6) follows. The congruence can be proved in the similar method of the proof of [6, Theorem 4] by congruences (4) and (5).

Q.E.D. 
Proposition 4. Let $m \geq 0, n \geq 0$ and $m+n=p-1$. Then

$$
b(m) \equiv(-1)^{m-1} b(n) \quad \bmod p .
$$

Proof. From the congruence (4), (5) and Proposition 3, $b(0) \equiv$ $-b(p-1) \equiv 0 \bmod p$. And by the definition of $b(n), \operatorname{ord}_{p} b(p) \geq 0$. Then $b(1) \equiv b(p-2) \equiv 5 \bmod p$ by the recurrence (6). By induction on $m$, similarly in Proposition 1, we can prove.

Q.E.D.

Theorem 3. Let $m \geq 0, n \geq 0$ and $m+n=p-1$. Then

$$
a(m) \equiv(-1)^{m}\{a(n)-p b(n)\} \quad \bmod p^{2} .
$$

Proof. It is clear from (4), (5) and Proposition 4 in the case of $m=$ 0, 1. From the recurrence (3), (6) and the congruence

$$
\begin{aligned}
(m+1)^{2} a(m+1) & \\
\equiv & \left\{11(p-m)^{2}-11(p-m)+3\right\} a(m)+(p-m)^{2} a(m-1) \\
& \quad-11 p\{2(p-m)-1\} a(m)-2 p(p-m) a(m-1) \quad \bmod p^{2},
\end{aligned}
$$

it can be also shown by inductive method.

Q.E.D.

\section{$\S 3$. Congruence of $c(n)$}

If $p \equiv 3 \bmod 4$, we can not obtain the congruence of $b((p-1) / 2)$ from Proposition 4. Therefore we prepare the numbers $c(n)$.

Let, for all odd numbers $n \geq 1$,

$$
c(n)=\sum_{k=1}^{n}\left(\begin{array}{l}
n \\
k
\end{array}\right)^{3}(-1)^{k}\left[\frac{3}{n-k+1}+\cdots+\frac{3}{n}\right] .
$$

Let $p$ be an odd prime. From the congruence

$$
\left(\begin{array}{c}
\frac{p-1}{2}+k \\
k
\end{array}\right) \equiv(-1)^{k}\left(\begin{array}{c}
\frac{p-1}{2} \\
k
\end{array}\right) \quad \bmod p
$$

and

$$
\frac{1}{\frac{p-1}{2}+k+1}+\cdots+\frac{1}{\frac{p-1}{2}}+\frac{1}{\frac{p+1}{2}}+\cdots+\frac{1}{\frac{p-1}{2}+k} \equiv 0 \bmod p
$$

where $1 \leq k \leq(p-1) / 2$, we have

$$
3 b\left(\frac{p-1}{2}\right) \equiv c\left(\frac{p-1}{2}\right) \quad \bmod p \quad \text { if } p \equiv 3 \bmod 4 \text {. }
$$


Proposition 5. The numbers $c(n)$ satisfy the recurrence

$$
n^{2} c(n)=-3\left\{9(n-1)^{2}-1\right\} c(n-2)
$$

for all odd numbers $n \geq 3$.

Proof. Let

$$
\begin{aligned}
f_{n}(k)= & 2\left(14 n^{2}+n-1\right)-3\left(26 n^{2}-n-3\right) k / n+3\left(29 n^{2}-3\right) k^{2} / n^{2} \\
& -3\left(15 n^{2}+2 n-1\right) k^{3} / n^{3}+3(3 n+1) k^{4} / n^{3} \\
g_{n}(k)= & 2(28 n+1)-3\left(26 n^{2}+3\right) k / n^{2}+18 k^{2} / n^{3} \\
& +3\left(15 n^{2}+14 n-3\right) k^{3} / n^{4}-9(2 n+1) k^{4} / n^{4}
\end{aligned}
$$

and

$$
C_{n, k}=\frac{3}{n-k+1}+\cdots+\frac{3}{n} .
$$

Then we have

$$
\begin{gathered}
(n+1)^{2}\left(\begin{array}{c}
n+1 \\
k
\end{array}\right)^{3} C_{n+1, k}+3\left(9 n^{2}-1\right)\left(\begin{array}{c}
n-1 \\
k
\end{array}\right)^{3} C_{n-1, k} \\
+2(n+1)\left(\begin{array}{c}
n+1 \\
k
\end{array}\right)^{3}+54 n\left(\begin{array}{c}
n-1 \\
k
\end{array}\right)^{3} \\
=f_{n}(k)\left(\begin{array}{c}
n \\
k
\end{array}\right)^{3} C_{n, k}+f_{n}(k-1)\left(\begin{array}{c}
n \\
k-1
\end{array}\right)^{3} C_{n, k-1} \\
+g_{n}(k)\left(\begin{array}{l}
n \\
k
\end{array}\right)^{3}+g_{n}(k-1)\left(\begin{array}{c}
n \\
k-1
\end{array}\right)^{3} .
\end{gathered}
$$

We multiply both sides by $(-1)^{k}$. Taking summation from 1 to $n+1$ on $k$,

$$
\begin{aligned}
& (n+1)^{2} c(n+1)+3\left(9 n^{2}-1\right) c(n-1) \\
& \quad+2(n+1) \sum_{k=0}^{n+1}\left(\begin{array}{c}
n+1 \\
k
\end{array}\right)^{3}(-1)^{k}+54 n \sum_{k=0}^{n-1}\left(\begin{array}{c}
n-1 \\
k
\end{array}\right)^{3}(-1)^{k}=0 .
\end{aligned}
$$

If $n \equiv 0 \bmod 2$, two latter summations are equal to 0 .

Q.E.D.

Remark. The numbers $c(n)$ satisfy the recurrence (8) if $n \equiv 1 \bmod 2$.

Proposition 6. Let $p \equiv 3 \bmod 4$ be a prime, we have

$$
c\left(\frac{p-1}{2}\right) \equiv 0 \quad \bmod p .
$$

Proof. It is trivial if $p=3$. If $p \equiv 7 \bmod 12$ then $(p+2) / 3$ is odd. By (7), we have 


$$
\left(\frac{p+2}{3}\right)^{2} c\left(\frac{p+2}{3}\right)+3\left\{9\left(\frac{p-1}{3}\right)^{2}-1\right\} c\left(\frac{p-4}{3}\right)=0
$$

Then $c((p+2) / 3) \equiv 0 \bmod p$. Hence, $c(n) \equiv 0 \bmod p$ for $(p+2) / 3 \leq n \leq$ $p-2$ and $n$ odd. If $p \equiv 11 \bmod 12$ then $(p+4) / 3$ is odd. Then it can be proved in the same way.

Q.E.D.

\section{§4. Proof of Theorem 2}

Beukers and Stienstra showed that the generating function of $a(n)$ is a holomorphic solution of the Picard-Fuchs equation associated to the family of elliptic curves. From this argument and the $\zeta$-function of a certain K3-surface, they proved Theorem 1 (see $[2,11]$ ). Moreover we know that the right hand side of (1) is equal to $\eta(4 z)^{6}$ with $q=e^{2 \pi i z}$, $\operatorname{Im}(z)>0$, where $\eta(z)=q^{1 / 24} \prod_{n=1}^{\infty}\left(1-q^{n}\right)$ is Dedekind's $\eta$-function. From the Jacobi-Macdonald formula, we see

$$
\lambda_{p}=\left\{\begin{array}{cl}
4 a^{2}-2 p & \text { if } p \equiv 1 \bmod 4 \text { and } p=a^{2}+b^{2}, \quad a \equiv 1 \bmod 2 \\
0 & \text { if } p \equiv 3 \bmod 4 .
\end{array}\right.
$$

Hence if $p \equiv 1 \bmod 4$ then $\lambda_{p} \neq 0 \bmod p$. According to Theorem $1, m=1$ and $r=1$ then $a((p-1) / 2) \equiv \lambda_{p} \neq 0 \bmod p$.

Let us prove Theorem 2 using congruences of $a(n), b(n), c(n)$, and Theorem 1.

If $p \equiv 1 \bmod 4$ then $\frac{p-1}{2}$ is even. From Proposition $4, b\left(\frac{p-1}{2}\right)$ $\equiv-b\left(\frac{p-1}{2}\right) \bmod p . \quad$ Hence $\quad b\left(\frac{p-1}{2}\right) \equiv 0 \bmod p . \quad$ Then $a\left(\frac{m p^{2}-1}{2}\right)$ $\equiv a\left(\frac{m p-1}{2}\right) a\left(\frac{p-1}{2}\right) \bmod p^{2} \quad$ and $\quad a\left(\frac{m p-1}{2}\right) \equiv a\left(\frac{m-1}{2}\right) a\left(\frac{p-1}{2}\right)$ $\bmod p^{2}$. Putting $r=2$ in Theorem $1, a\left(\frac{m p^{2}-1}{2}\right) \equiv \lambda_{p} a\left(\frac{m p-1}{2}\right) \bmod p^{2}$. Since $a\left(\frac{p-1}{2}\right) \neq 0 \bmod p$, this is reduced to $a\left(\frac{m p-1}{2}\right) \equiv \lambda_{p} a\left(\frac{m-1}{2}\right)$ $\bmod p^{2}$.

If $p \equiv 3 \bmod 4$ and $p \neq 3$ then

$$
a\left(\frac{p-1}{2}\right) \equiv \frac{p}{2} b\left(\frac{p-1}{2}\right) \equiv \frac{p}{6} c\left(\frac{p-1}{2}\right) \quad \bmod p^{2}
$$

by Theorem 3. From Proposition 6 , we have $a\left(\frac{p-1}{2}\right) \equiv 0 \bmod p^{2}$. Hence 


$$
a\left(\frac{m p-1}{2}\right) \equiv a\left(\frac{p-1}{2}\right) a\left(\frac{m-1}{2}\right) \equiv 0 \quad \bmod p^{2} .
$$

Thus we have completed the proof.

Q.E.D.

\section{§5. Application for other numbers}

Above method is applicable to other numbers which satisfy the relation such as (2) (see [11]), and super congruence of $u(n)$ is shown in [8]. i.e.

TheOREm 4. Let $p \geq 3$ be a prime, and write

$$
\sum_{n=1}^{\infty} \xi_{n} q^{n}=q \sum_{n=0}^{\infty}\left(1-q^{2 n}\right)^{4}\left(1-q^{4 n}\right)^{4} .
$$

If $u\left(\frac{p-1}{2}\right) \neq 0 \bmod p$ then

$$
u\left(\frac{p-1}{2}\right) \equiv \xi_{p} \quad \bmod p^{2}
$$

Moreover we cite another example in this section.

Let, for any $n \geq 0$,

$$
u(n)=(-1)^{n} \sum_{k=0}^{n}\left(\begin{array}{l}
n \\
k
\end{array}\right)^{3} .
$$

F. Beukers and J. Stientstra showed the following congruence in [11]. Let $p \geq 3$, and write

$$
\sum_{n=1}^{\infty} \gamma_{n} q^{n}=q \prod_{n=1}^{\infty}\left(1-q^{n}\right)^{2}\left(1-q^{2 n}\right)\left(1-q^{4 n}\right)\left(1-q^{8 n}\right)^{2} .
$$

Then, for $m, r \in N, m$ odd,

$$
v\left(\frac{m p^{r}-1}{2}\right)-r_{p} v\left(\frac{m p^{r-1}-1}{2}\right)+\left(\frac{-2}{p}\right) p^{2} v\left(\frac{m p^{r-2}-1}{2}\right) \equiv 0 \quad \bmod p^{r}
$$

where $\left(\frac{\cdot}{\cdot}\right)$ is the Jacobi-Legendre symbol.

The numbers $w(n)$ which is (differential) of $v(n)$ can be formulate to

$$
w(n)=3(-1)^{n} \sum_{k=1}^{n}\left(\begin{array}{l}
n \\
k
\end{array}\right)^{3}\left[\frac{1}{n-k+1}+\cdots+\frac{1}{n}\right] .
$$

And for all primes $p \geq 3, n \geq 0$ and $0 \leq m \leq p-1$, we have

$$
v(n p+m) \equiv\{v(m)+p n w(m)\} v(n) \quad \bmod p^{2} .
$$


Then $v\left(\frac{p-1}{2}\right)$ of $\bmod p^{2}$ is determined by our method if $\left(\frac{-2}{p}\right)=1$, that is

$$
v\left(\frac{p-1}{2}\right) \equiv \gamma_{p}+\frac{p}{2} w\left(\frac{p-1}{2}\right) \quad \bmod p^{2}
$$

\section{REFERENCES}

[1] F. Beukers, Irrationality of $\pi^{2}$, periods of an elliptic curve and $\Gamma_{1}(5)$, Progress in Math., 31, pp. 47-66, Birkhäuser, 1983.

[2] - Arithmetical properties of Picard-Fuchs equation, Progress in Math., 51, pp. 33-38, Birkhäuser, 1984.

[ 3 ] - Some congruence for Apéry numbers, J. Number Theory, 21 (1985), 141-155.

[4] - Another congruence for Apéry numbers, J. Number Theory, 25 (1987), 201210.

[5] S. Chowla, J. Cowles and M. Cowles, Congruence properties of Apéry numbers, J. Number Theory, 12 (1980), 188-190.

[ 6 ] I. Gessel, Some congruence for the Apéry numbers, J. Number Theory, 14 (1982), $362-368$.

[ 7 ] L. Van Hamme, Proof of a conjecture of Beukers on Apéry numbers, Proceedings of the conference on $p$-adic analysis, Hengelhoef, Belgium, 1986.

[ 8 ] T. Ishikawa, On Beukers' conjecture, Kobe J. Math., 6 (1989), 49-52.

[ 9 ] Y. Mimura, Congruence properties of Apéry numbers, J. Number Theory, 16 (1983), 138-146.

[10] A. J. van der Poorten, A proof that Euler missed … Apéry's proof of the irrationality of $\zeta(3)$, Math. Intelligencer, 1 (1979), 195-203.

[11] J. Stienstra and F. Beukers, On the Picard-Fuchs equation and the formal Brauer group of certain elliptic K3 surface, Math. Ann., 271 (1985), 269-304.

Department of Mathematics

Kobe University

Rokko, Kobe $65 \%$

Japan 